\title{
CLASSIFICAÇÃO DE BACIAS SEDIMENTARES: UMA REVISÃO COMENTADA
}

\author{
MARCELO A. MARTINS-NETO
}

\begin{abstract}
During the evolution of the knowledge regarding sedimentary basins, many authors have discussed criteria and proposed schemes for their classification, most of them driven by geotectonic theories like Geossinclinal and Plate Tectonics. Firstly, the present paper discusses the definition of a sedimentary basin, since there are two different approaches in the literature. The first is based on geodynamic criteria, where basins are defined by their linked subsidence mechanisms, related to a single tectonic regime or tectono-thermal event, responsible for the development of a first-order basin cycle. According to the second approach, a basin is defined as a region characterized by the accumulation of a thick sedimentary package during a long time span, regardless the existence of significative hiatuses and/or unconformities inside the package and the stacking of more than one first-order cycle. After a historical synthesis about the classification of sedimentary basins, the merits and efficiency of classification proposals are discussed, including the particular case of the intracratonic basins, as well as perspectives for the future. It is concluded that the basic classification criteria proposed by Dickinson (1974), although incomplete and/or deficient in some aspects, are very efficient for practical purposes, mostly by emphasizing the type of plate interaction (tectonic regime) during sedimentation (if divergent, convergent or strike-slip). The approach explaining the evolution of basins based on plate interactions solved the major problem of the previous classification schemes that was the proliferation of names for particular cases, as well as has direct implications in terms of stress field, structural and stratigraphic styles, being very efficient in the definition of the type of basin and having a predictive character in terms of processes and products.
\end{abstract}

Keywords: sedimentary basins, classification, basin analysis

Resumo Ao longo da evolução dos conhecimentos sobre bacias sedimentares, em função da necessidade intrínseca de se ordenar o conhecimento, vários autores se preocuparam em adotar critérios e propor esquemas para classificar as bacias sedimentares, na maior parte dos casos influenciados por teorias geotectônicas como Geossinclinal e Tectônica de Placas. Inicialmente, o artigo discute a definição de bacia sedimentar, uma vez que duas abordagens são encontradas na literatura, uma geodinâmica, onde bacias são definidas pela atuação de mecanismos de subsidência interligados, relativos ao mesmo regime tectônico ou evento tectonotermal, responsável pelo desenvolvimento de um ciclo de embaciamento de primeira ordem, e outra geográfica, que significa uma região caracterizada pela acumulação de uma pilha espessa de sedimentos por um longo período no tempo geológico, sem levar em consideração a existência de significativos hiatos e/ou discordâncias dentro do pacote e o empilhamento de mais de um ciclo de la ordem. Após uma síntese sobre a evolução dos conhecimentos acerca da classificação de bacias sedimentares, o artigo discute os méritos e a eficiência dos mesmos e de seus critérios norteadores, incluindo o caso particular das bacias intracratônicas, bem como discute perspectivas futuras do tema. Ao final, conclui que os critérios básicos propostos por Dickinson (1974), embora incompletos e/ou deficientes sobre alguns aspectos, se mostraram bastante eficientes do ponto de vista prático, sobretudo ao enfatizarem o tipo de interação de placas ativo (regime tectônico) durante a sedimentação (se divergente, convergente ou transcorrente). $O$ fato da evolução de bacias poder ser explicada com base nas relações de interação entre placas resolveu o problema maior dos esquemas anteriores, que era a proliferação de nomes para satisfazer casos particulares, bem como possui implicações diretas em termos de campos de tensão, estilos estruturais e estilos estratigráficos, conferindo uma eficiência prática na definição do tipo de bacia e um caráter preditivo em termos de processos e produtos.

Palavras-chave: bacias sedimentares, classificação, análise de bacias

INTRODUÇÃO Bacias sedimentares estão presentes em todos os continentes do planeta e o registro geológico mostra que elas existem desde os primórdios da evolução da Terra. Os greenstone belts arqueanos são bons exemplos (p. ex., $>3,7 \mathrm{Ga}$ Isua greenstone belt, Groelândia, Fedo et al. 2001), sem contar com as suítes gnáissicas paraderivadas mais antigas, embasamento dos greenstone belts, que, de forma indireta, atestam a ocorrência de bacias sedimentares ainda mais antigas.

Ao longo da evolução dos conhecimentos sobre bacias sedimentares, em função da necessidade intrínseca de se ordenar o conhecimento, vários autores se preocuparam em adotar critérios e propor esquemas para classificar as bacias sedimentares. É notável, como exposto a seguir, a influência das grandes teorias geotectônicas como elementos norteadores dos esquemas classificatórios, bem como, em tempos recentes, a adoção do paradigma de enfoque processual em substituição a uma base puramente descritiva.

Desta forma, este artigo tem por objetivo fazer uma revisão da evolução dos conhecimentos, bem como apresentar o estado da arte acerca da classificação de bacias sedimentares, tema im- portante para os estudiosos do assunto, sobretudo a geólogos de petróleo, uma vez que a definição de um tipo de bacia implica na predição de estilos estruturais, estilos estratigráficos e plays característicos.

DEFININDO UMA BACIA SEDIMENTAR Ocorrendo em diversos ambientes tectônicos, as bacias sedimentares estão sujeitas a diferentes mecanismos e regimes de subsidência, sendo que mais de um destes podem ocorrer simultaneamente ou suceder um ao outro serialmente, durante a evolução de uma bacia. Adotando-se uma concepção geodinâmica, uma bacia sedimentar é definida pela atuação de mecanismos de subsidência interligados, relativos ao mesmo regime tectônico ou evento tectono-termal, responsável pelo desenvolvimento de um ciclo de embaciamento de primeira ordem (" 1 st-order basin-fill cycle") (ver discussões em Allen \& Allen 1990, Eriksson et al. 2001a). O pacote sedimentar relativo a um ciclo de embaciamento pode então ser definido como uma seqüência de $1^{\text {a }}$ ordem (ou megasseqüência) limitada por discordâncias de primeira ordem. Assim, cada sequiência de $1^{\mathrm{a}}$ ordem pode ser reconhecida 
com base no seu arcabouço estratigráfico e na interpretação do seu regime tectônico e controles estratigráficos. Bacias como a Bacia de Campos (Dias et al. 1990) ou a Bacia Espinhaço (Martins-Neto 1998, 2000) são exemplos de definições adotando-se critérios geodinâmicos, uma vez que ambas representam o registro de processos tectono-termais contínuos e geneticamente interdependentes, nestes casos representados por uma fase rifte de estiramento crustal e subsidência mecânica e uma subseqüente fase flexural de re-equilíbrio termal (ver outros exemplos de aplicação desta concepção em Krapez 1993, Martins-Neto et al. 2001, Eriksson et al. 2001a, 2001b).

Bacias sedimentares têm sido, entretanto, definidas algumas vezes com base em uma concepção geográfica, que significa uma região caracterizada pela acumulação de uma pilha espessa de sedimentos por um longo período no tempo geológico, sem levar em consideração a existência de significativos hiatos e/ou discordâncias dentro do pacote, muitas vezes envolvendo um intervalo de tempo de algumas centenas de milhões de anos, e o empilhamento de mais de um ciclo de $1^{\text {a }}$ ordem. A Bacia do São Francisco é um bom exemplo de definição conforme uma concepção geográfica, uma vez que possui em seu registro uma sucessão de ciclos de $1^{a}$ ordem que incluem, por exemplo, margens passivas (seqüências Paranoá/Canastra e Macaúbas) e um ciclo de antepaís (Seqüência Bambuí), separados por discordâncias com lapsos de tempo da ordem de centenas de milhões de anos, que individualizam entidades com histórias geodinâmicas próprias e independentes (Martins-Neto \& Alkmim 2001, Martins-Neto 2005). A adoção de tal critério levou, como discutido mais à frente neste artigo, à definição da categoria "Bacias Intracratônicas", que, na maioria dos casos envolve mais de um ciclo de embaciamento de $1^{\text {a }}$ ordem.

\section{EVOLUÇÃO DOS ESQUEMAS CLASSIFICATÓRIOS}

\section{A era da Teoria Geossinclinal A pedra fundamental para} as primeiras iniciativas de classificação de bacias sedimentares foi lançada em 1859 pelo geólogo norte-americano James Hall, através da introdução das bases do conceito que viria a ser, posteriormente, denominado de geossinclinal por Dana em 1873. Segundo Hall (1859), que se dedicou ao estudo das sucessões paleozóicas que compõem a Cordilheira dos Apalaches, EUA, as montanhas se formariam através do dobramento de áreas caracterizadas pelo acumulo de significativos pacotes sedimentares, sendo a deposição constante de sedimentos e o peso destes responsáveis pelo "afundamento" contínuo do embasamento e pela preservação de espessas sucessões sedimentares. Ainda segundo este autor, os dobramentos e formação das montanhas seriam fruto de uma compressão associada à subsidência da pilha sedimentar. As bases da Teoria Geossinclinal foram ampliadas com as seguintes considerações de Dana, em 1866 e 1873: i) uma área topograficamente positiva, denominada de geoanticlinal, forneceria os sedimentos para a bacia geossinclinal adjacente; ii) os dobramentos e a formação de montanhas não seriam associados à subsidência, mas sucedia esta, introduzindo, assim, o conceito de fase orogenética; iii) propôs que o mecanismo motriz da geração de bacias sedimentares e sua posterior deformação seriam esforços tangenciais associados à contração da Terra, fruto de seu resfriamento; iv) finalmente, Dana reconheceu diferentes províncias paleogeográficas (geoanticlinal, geossinclinal e oceano) com importantíssimas implicações para a evolução dos conceitos geológicos, uma vez que atribuiu um padrão evolutivo onde geossinclinais, por compressão e dobramento, se tornavam cadeias de montanhas e eram "soldados" marginalmente aos continentes, lançando o conceito de acresção continental.

Já na virada para o século 20, em 1900, E. Haug, observando que alguns geossinclinais possuíam depósitos de águas profun- das, considerou em primeira mão a relação entre taxas de subsidência e suprimento sedimentar. Onde a primeira suplanta a segunda teríamos os geossinclinais de água profunda e, no caso oposto, teríamos o assoreamento do geossinclinal.

Em 1940, o geólogo alemão Hans Stille propôs a primeira classificação formal dos geossinclinais (Tabela 1). Na discussão dos processos geológicos relacionados à evolução dos geossinclinais, Stille apontou a existência de geossinclinais que nunca teriam sido afetados por dobramentos e, conseqüentemente, não deram origem a cadeia de montanhas, bem como apresentou uma evolução cronológica para o magmatismo associado à evolução dos geossinclinais, separando os eventos pré-, sin- e pós-orogenéticos. Merece destaque a introdução e definição do termo ofiolito para um tipo de magmatismo pré-orogenético, caracterizado, segundo Stille (1940), por grandes derrames submarinos de natureza basáltica intercalados com os sedimentos e associados a gabros e peridotitos intrusivos. O magmatismo associado à evolução dos geossinclinais permitiria ainda, segundo Stille (1940), a definição de uma zona interna (internide), área de ocorrência destes, e de uma zona externa, sem magmatismo, denominada de externide. Ambos construíam os geossinclinais dobrados, geradores de montanhas, também denominados por Stille de Cadeias Alpinótipas. À área continental adjacente aos geossinclinais dobrados, construída em parte pela acresção destes, Stille deu o nome de craton. Nos crátons desenvolveriam-se as Cadeias Germanótipas que, segundo Stille, eram caracterizadas por blocos falhados e dobras associadas a estes. Com base no exposto acima, Stille erigiu em 1940 sua classificação (Tabela 1), dividida em dois grupos básicos: os ortogeossinclinais, com estilo alpinótipo e geradores de cadeias de montanhas; e os parageossinclinais, com estrutura germanótipa, desenvolvidos nos crátons. Adicionalmente, os ortogeossinclinais poderiam ser divididos em eugeossinclinais, englobando as internides, mais deformadas, e miogeossinclinais, menos deformados representavam as externides.

O próximo passo seria dado em 1947 e 1951 pelo norte-americano Marshall Kay, através de publicações que sintetizavam sua pesquisa (Kay, 1942, 1943, 1945a, 1945b; in Kay, 1947, 1951), realizadas a partir da proposição de Stille e tendo como palco o continente norte-americano. Kay $(1947,1951)$ manteve a subdivisão dos ortogeossinclinais em eu- e miogeossinclinais, apontando para a existência de sucessivas fases deformacionais, o que requereria uma acurada restauração paleogeográfica para uma interpretação segura. Para os parageossinclinais, de ambiência cratônica, Kay $(1947,1951)$ teceu uma série de considerações sedimentares, tais como sistemas deposicionais típicos e características das áreas de proveniência de sedimentos, e cunhou uma série de nomes para designar posicionamento dentro do cráton e/ou processos tectônicos envolvidos (Tabela 2).

Ressaltando a dificuldade de se erigir um esquema classificatório "perfeito" para bacias sedimentares, que satisfizesse todas as possibilidades de gênese, posição relativa no arcabouço crustal, grau de mobilidade, fonte e fácies de sedimentos, Weeks (1952) propôs nova classificação para "Geossinclinais ou Bacias Sedimentares" (Tabela 3), a qual, segundo o próprio autor, seria simples, completa e útil para todos os fins, sobretudo para o geólogo de petróleo.

A era da Tectônica de Placas Na década de 60 começaram a surgir trabalhos aplicando a Teoria da Tectônica de Placas ao estudo da evolução de bacias sedimentares, mesmo que ainda atados a conceitos e terminologias da Teoria Geossinclinal ( $p$. ex., Dietz 1961, 1963, Hsü 1965, Drake 1966, Laughton 1966, Menard 1967). Dewey (1969) e Mitchell \& Reading (1969), embora sem proporem formalmente uma classificação para bacias à luz da nova teoria, deram uma conotação genética a termos 
Tabela 1: Classificação de geossinclinais, segundo Stille (1940).

\begin{tabular}{|c|c|c|}
\hline \multirow{2}{*}{$\begin{array}{c}\text { ORTOGEOSSINCLINAIS } \\
\text { (Estruturas Alpinótipas) }\end{array}$} & $\begin{array}{c}\text { Eugeossinclinais } \\
\text { (internides) }\end{array}$ & $\begin{array}{c}\text { - orogênese primária } \\
\text { deformação tipificada por intensos dobramentos } \\
\text { magmatismo associado }\end{array}$ \\
\cline { 2 - 3 } & $\begin{array}{c}\text { Miogeossinclinais } \\
\text { (externides) }\end{array}$ & $\begin{array}{c}\text { orogênese secundária } \\
\text { sem magmatismo }\end{array}$ \\
\hline $\begin{array}{c}\text { PARAGEOSSINCLINAIS } \\
\text { (Cratônicos) }\end{array}$ & $\begin{array}{c}\text { Estruturas } \\
\text { Germanótipas }\end{array}$ & $\begin{array}{c}\text { deformação restrita, tipificada por blocos falhados e } \\
\text { dobras associadas a falhas } \\
\text { horsts e grabens }\end{array}$ \\
& $\begin{array}{c}\text { Estruturas } \\
\text { maciços, soergumentos e fossas }\end{array}$ \\
\hline
\end{tabular}

Tabela 2: Classificação de geossinclinais proposta por Kay (1947).

\begin{tabular}{|c|c|}
\hline \multirow{2}{*}{ ORTOGEOSSINCLINAIS } & Eugeossinclinais \\
\hline & Miogeossinclinais \\
\hline \multirow{5}{*}{ PARAGEOSSINCLINAIS } & $\begin{array}{l}\text { Exogeossinclinais e Autogeossinclinais: } \\
\text { na margem do cráton com proveniência do ortogeossinclinal }\end{array}$ \\
\hline & Zeugogeossinclinais: na margem do cráton com proveniência deste \\
\hline & Tafrogeossinclinais: limitado por falhas de alto ângulo \\
\hline & $\begin{array}{l}\text { Epieugeossinclinais e Idiogeossinclinais: } \\
\text { elongados e desenvolvidos sobre antigos geossinclinais }\end{array}$ \\
\hline & $\begin{array}{l}\text { Paraliogeossinclinais: } \\
\text { geossinclinais costeiros ao longo da margem continental }\end{array}$ \\
\hline
\end{tabular}

Tabela 3: Classificação de "geossinclinais ou bacias sedimentares", conforme Weeks (1952).

\begin{tabular}{|c|c|c|}
\hline GRUPO & \multicolumn{2}{|c|}{ CLASSE E SUB-CLASSE } \\
\hline \multirow{5}{*}{$\begin{array}{l}\text { FAIXAS } \\
\text { MóVEIS }\end{array}$} & \multirow{2}{*}{$\begin{array}{c}\text { MARGINAL } \\
\text { (EXTRA-CONTINENTAL) }\end{array}$} & marginal aberta \\
\hline & & marginal fechada \\
\hline & \multicolumn{2}{|c|}{ INTRA-CONTINENTAL } \\
\hline & INTERMONTANA & \multirow{2}{*}{$\begin{array}{l}\text { bacias de segunda ordem desenvolvidas } \\
\text { em montanhas ou faixas móveis }\end{array}$} \\
\hline & MEDIANA (INTERMONTANA) & \\
\hline \multirow{5}{*}{$\begin{array}{l}\text { REGIỐES } \\
\text { ESTÁVEIS }\end{array}$} & \multicolumn{2}{|c|}{ PLATAFORMA MARGINAL } \\
\hline & \multicolumn{2}{|c|}{ INTERIOR (INTRACRATÔNICA) PRÓXIMO } \\
\hline & \multicolumn{2}{|c|}{ INTERIOR (INTRACRATÔNICA) REMOTO } \\
\hline & \multicolumn{2}{|c|}{ GRABEN OU MEIO-GRABEN } \\
\hline & \multicolumn{2}{|c|}{ COSTEIRA ESTÁVEL } \\
\hline
\end{tabular}
to relativo de placas litosféricas, relação litosfera continental/ oceânica e criação de assoalho oceânico. Mitchell \& Reading (1969) dividiram os "geossinclinais" em quatro tipos: Atlânti- 
co, Andino, Arco de Ilha e Mar do Japão. Descreveram, para cada tipo, suas características deposicionais, natureza do embasamento (oceânico ou continental) e relação com espalhamento oceânico/subducção. Além disso, estes autores discorreram sobre mudanças de um tipo para outro de geossinclinal, bem como apresentaram e caracterizaram duas possibilidades para a orogenia associada os geossinclinais: tipo Andino ou Arco de Ilha e tipo Himalaiano ou de Colisão Continental.

Dewey \& Bird (1970), considerando que a classificação de Kay (1947) poderia ser adaptada com base no arcabouço da expansão/contração oceânica por acresção/consumo de litosfera, propuseram novo esquema "híbrido" para a classificação de bacias sedimentares (Tabela 4), atribuindo conceitos da Tectônica de Placas aos termos da classificação de Kay (1947).

Em três importantes artigos publicados em 1971, W.R. Dickinson afirmou que a Teoria da Tectônica de Placas poderia explicar qualquer tipo de bacia sedimentar e cinturões de montanhas, destacando que a antiga e, segundo o autor, ultrapassada Teoria Geossinclinal Clássica deveria ser abandonada, argumentando que "embora seus principios tenham desempenhado um importante papel na classificação de elementos tectônicos, eles são agora impedimentos desnecessários para se pensar claramente no futuro". Adicionalmente, importantes termos/concei- tos tais como "sistema arco/fossa" e "ambientes de antearco ou retroarco" são introduzidos nestes trabalhos.

Hsü (1972, in Bally \& Snelson, 1980) apontou uma série de deficiências e incongruências da Teoria Geossinclinal e suas classificações, quando analisadas à luz da Tectônica de Placas, destacando-se: i) O conceito de geossinclinal e as classificações relativas a ele foram baseados amplamente em deduções derivadas de observações geológicas de campo, principalmente reconstruções paleogeográficas muitas vezes difíceis de conciliar com exemplos de bacias atuais. Adicionalmente, estes trabalhos foram desenvolvidos antes de levantamentos oceanográficos e geofísicos, sobretudo sísmica de reflexão, mostrando-se conflitantes com as evidências destes. ii) Os geossinclinais descritos na literatura, quase na sua totalidade, foram deformados ou completamente modificados por dobramento ou processos erosivos, sendo eles atualmente remanescentes incompletos de bacias antigas. As classificações baseadas na Teoria Geossinclinal não diferenciam processos formadores dos modificadores e dependem da arriscada reconstituição de complexos dobrados, não permitindo a separação de aspectos factuais dos especulativos.

Em 1974, em um dos mais importantes e marcantes trabalhos sobre bacias sedimentares, W.R. Dickinson apresentou a primeira classificação baseada inteiramente na Tectônica de

Tabela 4: Classificação de geossinclinais, conforme Dewey \& Bird (1970).

\begin{tabular}{|c|c|c|c|}
\hline \multicolumn{3}{|r|}{ TERMINOLOGIA } & INTERPRETAÇÃO \\
\hline \multicolumn{3}{|c|}{ AUTOGEOSSINCLINAL } & bacia intracratônica não-linear \\
\hline \multirow{8}{*}{ 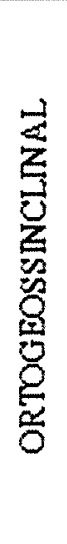 } & \multicolumn{2}{|r|}{ EPIEUGEOSSINCLINAI } & calha limitada por falha, desenvolvida durante distensão continental \\
\hline & \multicolumn{2}{|r|}{ MIOGEOSSINCLINAL } & calha adjacente à margem continental \\
\hline & \multicolumn{2}{|r|}{ MOGEOCLINAL } & $\begin{array}{c}\text { cunha que se espessa em direção ao oceano, adjacente à margem } \\
\text { continental }\end{array}$ \\
\hline & \multirow{5}{*}{ 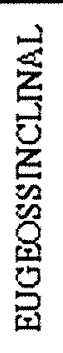 } & EPIEUGEOSSINCLINAL & como acima (= tafrogeossinclinal) \\
\hline & & PARALIOGEOSSINCLINAL & cunha espessa construida a partir da margem continental \\
\hline & & \multirow{2}{*}{ LEPTOGEOSSINCLINAL } & sopé continental faminto \\
\hline & & & planicie abissal oceânica, cordilheira meso-oceânica e montes submarinos \\
\hline & & KINEGEOCLINAL & $\begin{array}{c}\text { fossa ou complexo de fossa em contração entre a margem continental e } \\
\text { orógeno em desenvolvimento }\end{array}$ \\
\hline \multicolumn{3}{|c|}{ EXOGEOSSINCLINAI } & $\begin{array}{l}\text { fossa ou complexo de fossa desenvolvido em local de antigo } \\
\text { miogeossinclinal ou miogeoclinal }\end{array}$ \\
\hline \multicolumn{3}{|c|}{ ZEUGOGEOSSINCLINAL } & calha limitada por falha dentro de orógeno \\
\hline \multirow{7}{*}{ 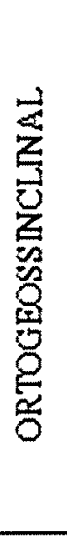 } & \multirow{7}{*}{ 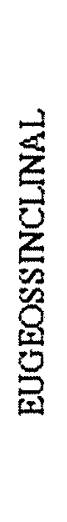 } & \multirow{2}{*}{ DIOGEOSSINCLINAI } & $\begin{array}{l}\text { bacia oceânica, restrita e estática, desenvolvida entre a margem continental } \\
\text { e o arco de itha }\end{array}$ \\
\hline & & & bacia oceânica restrita, desenvolvida entre arcos de itha \\
\hline & & ARCO DE ILHAS & $\begin{array}{c}\text { orógeno ensimático desenvolvido por acresção de sedimentos oceânicos, } \\
\text { vulcanismo, deformação e metamorfismo }\end{array}$ \\
\hline & & FOSSA OCEANICA & situada na zona de consumo de placas \\
\hline & & LEPTOGEOSSINCLINAL & planicie abissal oceânica, cordilheira meso-oceânica e montes submarinos \\
\hline & & \multirow{2}{*}{$\begin{array}{l}\text { BACIA OCEÂNICA } \\
\text { RESTRITA }\end{array}$} & $\begin{array}{c}\text { bacia oceânica em fase final de contração por consumo de placas em } \\
\text { margens em colisão }\end{array}$ \\
\hline & & & $\begin{array}{l}\text { bacia oceânica em fase final de contração por consumo de placas } \\
\text { envolvendo apenas uma margem }\end{array}$ \\
\hline \multicolumn{3}{|c|}{ EXOGEOSSINCLINAL } & fossa exterior (molassa) \\
\hline
\end{tabular}


Placas (Tabela 5). Dickinson (1974) afirmou que a ambiência tectônica das bacias pode ser descrita com base em três fatores fundamentais: (i) o tipo de crosta/litosfera que serve de substrato para a bacia (continental, oceânica ou transicional), (ii) a proximidade da bacia em relação à margem da placa (intraplaca ou zonas de interação de placas) e (iii) o tipo de junção ou junções de placas associada à bacia (divergente, convergente ou transformante). Em relação ao último fator, o autor afirmou que os três tipos de junções são end members e que variações destes são representadas por relações oblíquas. Cabe ressaltar que o caráter evolutivo e seqüencial das bacias controlado por processos tectônicos envolvendo o mudanças de situação em relação às placas e da interação destas, bem como a concepção de bacias sucessoras é amplamente discutido no trabalho. Dickinson (1974) cita a existência de trends evolutivos típicos, destacando, por exemplo, o ciclo de abertura e fechamento de oceanos com rifteamento inicial, fase de proto-oceano, ruptura continental e formação de bacia oceânica, inversão de movimento, instalação de sistema de arco/fossa/zona de subducção, colisão continental, geração de sutura e desenvolvimento de bacia periférica associada no antepaís. Desta forma, os parâmetros que orientam a classificação das bacias (i, ii e iii acima) podem ser arranjados de forma variada no tempo e no espaço, de modo a satisfazer o espectro existente de bacias. Adicionalmente, Dickinson (1974) relacionou mecanismos de subsidência à formação e evolução das bacias, bem como discutiu aspectos da relação entre tectônica, sedimentação e magmatismo para cada categoria.

Em 1980, após muitos avanços conceituais ocorridos durante a década de 70 , surgiram na literatura duas novas proposições de classificação de bacias sedimentares. A primeira, de Bally \& Snelson (1980) no clássico artigo "Realms of Subsidence", mantinha como critério os princípios de Dickinson (1974) envolvendo tipo de litosfera e posicionamento em relação à(s) placa(s) envolvida(s) (Tabela 6). Os autores apresentaram, adicionalmente, uma discussão enfocando o potencial petrolífero de cada categoria de bacias. A segunda, de Klemme (1980), por ter sido feita direcionada à indústria do petróleo, incorporou, além dos critérios usuais ligados à Tectônica de Placas, elementos associados à ocorrência de petróleo (Tabela 7). Embora Dickinson (1974) tenha explicitado a interação transcorrente entre placas, apenas em 1982 as bacias associadas a sistemas transcorrentes ou transformantes foram incorporadas aos esquemas classificatórios (Reading, 1982, in Allen \& Allen, 1990). Cabe ressaltar que Klemme (1980) considerou seu Tipo 5 - Bacias Pull-apart como uma derivação evolutiva de bacias tipo rifte, embora o termo tenha sido consagrado depois para designar bacias desenvolvidas em sistemas transcorrentes.

Kingston et al. (1983) propuseram um esquema alternativo para classificação de bacias, baseado na tectônica formadora da bacia, nas suas seqüências deposicionais e na tectônica modificadora da bacia (Fig. 1). O esquema baseia-se na atribuição de códigos para cada tipo de ciclo tectono-sedimentar, prevendo a existência de bacias policíclicas, às quais uma sequiência adequada de códigos constituiria uma fórmula que ilustraria a história evolutiva da bacia. Foi também, em parte, erigido buscando-se praticidade e adequação à informatização crescente na época de seu desenvolvimento e lançamento. O esquema de Kingston et al. (1983) foi por um período tentativamente aplicado (p.ex., Figueiredo \& Raja Gabaglia, 1986, para as principais bacias fanerozóicas brasileiras), mas, ao mostrar-se complexo e, sobretudo, ao requerer uma familiaridade com sua gama de códigos, não criou raízes dentro dos analistas de bacias.

Miall $(1984,1990)$, baseando-se nos critérios de Dickinson (1974) e em conceitos desenvolvidos desde então, buscou aperfeiçoar o esquema de Dickinson para classificar bacias sedimentares (Tabela 8).

A era moderna A disponibilidade e/ou operacionalidade de novas ferramentas (p.ex., modelagem computacional e sísmica de reflexão profunda) ou o significativo aperfeiçoamento de outras (p.ex., processamento sísmico) levou, durante os anos 80 , a um grande salto no entendimento da evolução de bacias sedimentares, incorporando novas tendências aos esquemas classificatórios. Nesta década, por exemplo, merecem destaque significativos avanços no entendimento de aspectos reológicos da litosfera e nos mecanismos e regimes de subsidência.

Baseando-se nas premissas e no esquema classificatório de Dickinson (1974), Ingersoll (1988) propôs um novo esquema, que foi aperfeiçoado posteriormente por Ingersoll \& Busby (1995) (Tabela 9). Ingersoll (1988), levando em conta a complexidade da evolução da maioria das bacias (prođuto de uma sucessão contínua de diferentes processos tectônicos em diferentes tipos de interação entre placas), destacou a importância da utilização dos termos referentes à interação entre placas (divergente, convergente, transformante), ao invés de termos que enfatizam, por exemplo, o campo de tensões dominante (distensivo, compressivo e transcorrente), ou termos como "margens ativas ou passivas". Ingersoll \& Busby (1995) apontaram

Tabela 5: Classificação de bacias, conforme Dickinson (1974).

\begin{tabular}{|c|c|c|}
\hline \multirow{3}{*}{ INTRAPLACA } & \multicolumn{2}{|c|}{ substrato continental (intracontinentais, riftes abortados e aulacógenos) } \\
\hline & \multicolumn{2}{|r|}{ substrato oceânico (bacias oceânicas profundas) } \\
\hline & \multicolumn{2}{|c|}{ substrato transicional (herdado de antigas junções de placas) } \\
\hline \multirow{7}{*}{$\begin{array}{c}\text { ZONAS DE } \\
\text { INTERAÇÃO } \\
\text { ENTRE PLACAS }\end{array}$} & \multirow{2}{*}{ DIVERGENTE } & margens rifteadas \\
\hline & & proto-oceano e oceano aberto em dorsais \\
\hline & \multirow[t]{2}{*}{ CONVERGENTE } & $\begin{array}{c}\text { sistema arco/fossa (bacias de fossa, antearco, intra-arco, inter-arco, } \\
\text { retroarco, foreland de retroarco) }\end{array}$ \\
\hline & & bacias foreland periféricas a colisões continentais \\
\hline & \multirow{3}{*}{ TRANSFORMANTE } & continental - continental \\
\hline & & oceânica - oceânica \\
\hline & & continental - oceânica \\
\hline
\end{tabular}


Tabela 6: Classificação de bacias proposta por Bally \& Snelson (1980).

\begin{tabular}{|c|c|c|}
\hline \multirow{2}{*}{$\begin{array}{l}\text { BACIAS LOCALIZADAS SOBRE } \\
\text { LITOSFERA RÍGIDA, NÃO } \\
\text { ASSOCIADAS A FORMAÇÄO DE } \\
\text { MEGASSUTURAS }\end{array}$} & $\begin{array}{l}\text { RELACIONADAS À FORMAÇÃO } \\
\text { DE CROSTA OCEÂNICA }\end{array}$ & $\begin{array}{c}\text { - riftes } \\
\text { - bacias associadas a falhas transformantes oceânicas } \\
\text { - planicies abissais oceânicas } \\
\text { - margens passivas do tipo Atlântico }\end{array}$ \\
\hline & $\begin{array}{l}\text { LOCALIZADAS SOBRE } \\
\text { LITOSFERA CONTINENTAL PRE- } \\
\text { MESOZOICA }\end{array}$ & bacias cratônicas \\
\hline \multirow{3}{*}{$\begin{array}{l}\text { BACIAS PERISSUTURAIS SOBRE } \\
\text { LITOSFERA RÍGIDA, ASSOCIADAS À } \\
\text { FORMAÇÄO DE MEGASSUTURA } \\
\text { COMPRESSIONAL }\end{array}$} & \multicolumn{2}{|c|}{$\begin{array}{l}\text { FOSSA MARINHA PROFUNDA SOBRE CORSTA OCEÂNICA, ADJACENTE A } \\
\text { MARGEM DE SUBDUCÇÃO DO TIPO B }\end{array}$} \\
\hline & \multicolumn{2}{|c|}{$\begin{array}{l}\text { ANTEFOSSA E SEDIMENTOS PLATAFORMAIS SOBRE CROSTA CONTINENTAL, } \\
\text { ADJACENTE A MARGEM DE SUBDUCÇÃO DO TIPO A }\end{array}$} \\
\hline & \multicolumn{2}{|c|}{$\begin{array}{l}\text { BACIAS DO "TIPO CHINÊS" ASSOCIADAS A BLOCOS FALHADOS DISTAIS } \\
\text { RELACIONADOS A MEGASSUTURA COMPRESSIONAL, NÃO ASSOCIADAS A } \\
\text { MARGEM DE SUBDUCÇÃO DO TIPO A }\end{array}$} \\
\hline \multirow{3}{*}{$\begin{array}{l}\text { BACIAS EPISSUTURAIS } \\
\text { LOCALIZADAS EM MEGASSUTURAS } \\
\text { COMPRESSIONAIS }\end{array}$} & $\begin{array}{l}\text { ASSOCIADAS A ZONAS DE } \\
\text { SUBDUCÇÃO DO TIPOB }\end{array}$ & $\begin{array}{l}\text { - bacias de antearco } \\
\text { - bacias de retroaco circumpacificas }\end{array}$ \\
\hline & $\begin{array}{l}\text { BACIAS DE RETROARCO } \\
\text { ASSOCIADAS A COLISẪO } \\
\text { CONTINENTAL (SUBDUCÇÂOO DO } \\
\text { TIPO A) }\end{array}$ & $\begin{array}{c}\text { - bacias do tipo Panoniana (sobre crosta continental) } \\
\text { - bacias do tipo Oeste-mediterrânea (sobre crosta } \\
\text { transicional ou oceânica) }\end{array}$ \\
\hline & $\begin{array}{l}\text { BACIAS RELACIONADAS A } \\
\text { SISTEMAS DE } \\
\text { MEGACISALHAMENTO } \\
\text { EPISSUTURAL }\end{array}$ & $\begin{array}{l}\text { - bacias do tipo Great basin } \\
\text { - bacias do tipo Califórnia }\end{array}$ \\
\hline
\end{tabular}

Tabela 7: Classificação de bacias proposta por Klemme (1980).

\begin{tabular}{|c|c|c|c|}
\hline \multirow{3}{*}{ 悹密 } & \multirow{4}{*}{ 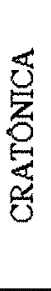 } & \multicolumn{2}{|r|}{ TIPO 1 - INTERIOR SIMPLES } \\
\hline & & \multicolumn{2}{|r|}{ TIPO 2 - COMPOSTA } \\
\hline & & \multicolumn{2}{|r|}{ TIPO 3 - RIFTE } \\
\hline \multirow{4}{*}{ 胥 } & & \multicolumn{2}{|c|}{ TIPO 4-ÄREAS DEPRIMIDAS (DOWNWARP) EM PEQUENAS BACIAS OCEÂNICAS } \\
\hline & \multirow{4}{*}{ 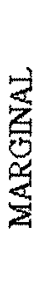 } & \multicolumn{2}{|r|}{ TIPO 5-BACIAS "PULL-APART" } \\
\hline & & \multirow[t]{2}{*}{ INTERMONTANA } & $\begin{array}{c}\text { TIPO 6 - BACIAS ASSOCIADAS A ZONAS DE SUBDUCÇÃO (ANTEARCO, } \\
\text { RETROARCO E NÃO-ARCO) }\end{array}$ \\
\hline & & & TIPO 7 - BACIAS MEDIANAS \\
\hline$?$ & & \multicolumn{2}{|r|}{ TIPO 8 - DELTAS } \\
\hline
\end{tabular}

a necessidade da classificação de bacias de acordo com sua ambiência tectônica sindeposicional, enfatizando a colocação de Dickinson (1974): “A evolução de uma bacia sedimentar pode ser vista como o resultado de uma sucessão de ambientes tectônicos distintos com diferentes padrões de interação entre placas, cujos efeitos se somam em um processo evolutivo contínuo". Ingersoll \& Busby (1995) inovaram em relação à proposta de Ingersoll (1988) ao distinguirem os ambientes intraplaca e divergentes. Entretanto, fica claro que este esquema peca em criar um número excessivo de categorias para classificar estágios de uma evolução contínua ou para satisfazer casos particulares. Por exemplo, as "margens continentais rifteadas" do tipo atlântico, embora vistas com uma etapa evolutiva madura do processo de rifteamento e quebra de continentes, são subdivididas em duas categorias: (i) estágio terraço e sopé continentais, que englobaria os depósitos de plataforma, talude e sopé continentais, já em condições de oceano aberto, porém relativos a fases iniciais com subsidência predominantemente devida à sobrecarga sedimentar; e (ii) estágio prismas continentais, alcançado apenas onde o suprimento sedimentar é volumoso o suficiente para causar progradação da linha de costa sobre crosta oceânica (Ingersoll, 1988). Uma categoria ("ilhas oceânicas, cristas e platôs") foi 


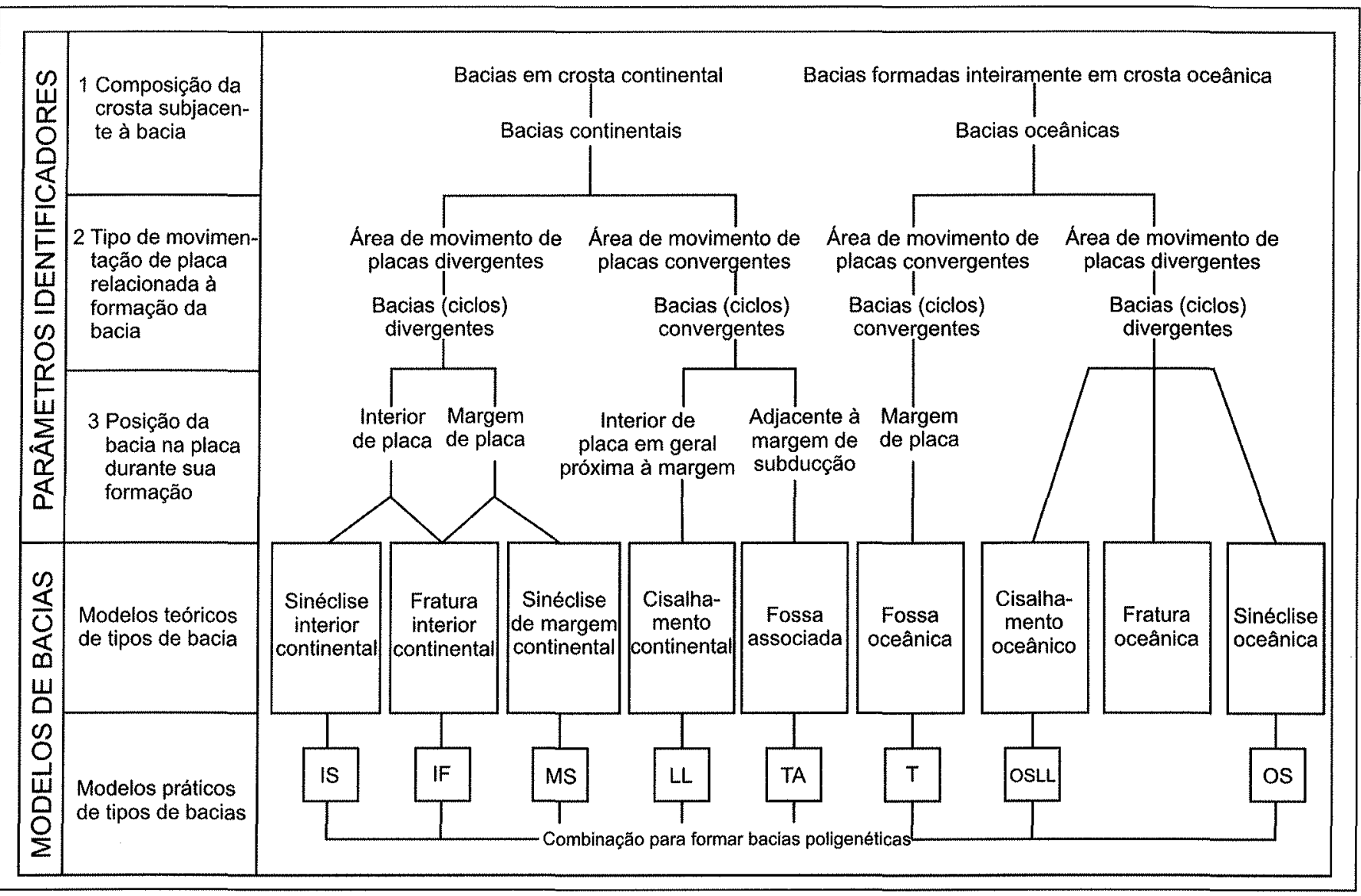

Figura 1: Esquema de classificação de bacias de Kingston et al. (1983). Versão em português obtida de Figueiredo \& Raja Gabaglia (1986).

Tabela 8: Classificação de bacias conforme Miall (1984, 1990).

\begin{tabular}{|c|c|}
\hline \multirow{4}{*}{$\begin{array}{l}\text { BACIAS DE MARGEM } \\
\text { DIVERGENTE }\end{array}$} & bacias rifte (bacias de arqueamento rifteado, marginais de arqueamento, sag, meio-graben) \\
\hline & bacias marginais oceânicas (jovens, tipo Mar Vermelho e maturas tipo Atlântico) \\
\hline & aulacógenos e riftes abortados \\
\hline & ilhas oceânicas, montes submarinos e platôs \\
\hline \multirow{4}{*}{$\begin{array}{l}\text { BACIAS DE MARGEM } \\
\text { CONVERGENTE }\end{array}$} & fossas e complexos de subducção \\
\hline & bacias de antearco \\
\hline & bacias de inter-arco e retroarco \\
\hline & bacias foreland de retroarco \\
\hline \multirow{2}{*}{$\begin{array}{l}\text { BACIAS ASSOCIADAS A } \\
\text { FALHAS } \\
\text { TRANSFORMANTES E } \\
\text { TRANSCORRENTES }\end{array}$} & $\begin{array}{c}\text { localização da bacia: falhas transformantes de borda de placa, falhas transformantes em } \\
\text { margem divergente, falha transcorrente em margem convergente, falha transcorrente em zona } \\
\text { de sutura }\end{array}$ \\
\hline & $\begin{array}{l}\text { tipo de bacia: bacias em sistemas de falhas entrelaçadas, bacias de terminação de falhas, bacias } \\
\text { pull-apart em sistemas de falha en echelon, bacias transrotacionais }\end{array}$ \\
\hline \multirow{3}{*}{$\begin{array}{c}\text { BACIAS } \\
\text { DESENVOLVIDAS } \\
\text { DURANTE COLISÃO } \\
\text { CONTINENTAL E } \\
\text { FORMAÇÃO DE } \\
\text { SUTURAS }\end{array}$} & bacia foreland ou foredeep periférica (sobre placa cavalgada) \\
\hline & bacias em calhas intra-sutura (bacias oceânicas remanescentes) \\
\hline & bacias hinterland foreland, transcorrentes e grabens (sobre placa cavalgante) \\
\hline & BACIAS CRATÔNICAS \\
\hline
\end{tabular}


criada, por exemplo, para englobar a acumulação de sedimentos em feições fisiográficas particulares.

Allen \& Allen (1990), após incorporar conceitos tectonofísicos desenvolvidos sobretudo nos anos 80 e apontando que as classificações anteriores pecam em não mostrar claramente as similaridades e diferenças essenciais entre bacias, do ponto de vista de mecanismos litosféricos, propuseram que a classificação de bacias deveria levar em consideração tais mecanis-

Tabela 9: Classificação de bacias conforme Ingersoll (1988) e Ingersoll \& Busby (1995).

\begin{tabular}{|c|c|c|}
\hline \multirow{2}{*}{$\begin{array}{l}\text { AMBIENTE } \\
\text { DIVERGENTE }\end{array}$} & riftes continentais & riftes em crosta continental, comumente associados com vulcan ismo bimodal \\
\hline & calhas proto-oceânicas & $\begin{array}{l}\text { bacias oceânicas incipientes com crosta oceânica juvenil, flanqueadas por } \\
\text { margens continentais rifteadas }\end{array}$ \\
\hline \multirow{7}{*}{$\begin{array}{l}\text { AMBIENTE } \\
\text { INTRAPLACA }\end{array}$} & terraço e sopé continentais & $\begin{array}{c}\text { margens rifteadas maduras em ambiente intraplaca, na interface continental- } \\
\text { oceânica }\end{array}$ \\
\hline & prismas continentais & $\begin{array}{l}\text { cunhas sedimentares progradacionais construídas sobre bordas de margens } \\
\text { rifteadas }\end{array}$ \\
\hline & bacias intracratônicas & bacias cratônicas amplas desenvolvidas sobre riftes fósseis axiais \\
\hline & $\begin{array}{l}\text { bacias cratônicas } \\
\text { plataformais }\end{array}$ & $\begin{array}{c}\text { pacotes sedimentares pouco espessos e lateralmente contínuos, cobrindo } \\
\text { crátons estáveis }\end{array}$ \\
\hline & bacias oceânicas ativas & $\begin{array}{l}\text { bacias sobre crosta oceânica formadas em bordas divergentes de placas, não } \\
\text { relativas a sistemas de arco-fossa (espalhamento oceânico ainda ativo) }\end{array}$ \\
\hline & $\begin{array}{l}\text { ilhas oceânicas, cristas e } \\
\text { platôs assísmicos }\end{array}$ & $\begin{array}{l}\text { prismas e coberturas sedimentares em ambiente intraoceânico, excetuando } \\
\text { arcos magmáticos }\end{array}$ \\
\hline & bacias oceânicas inativas & $\begin{array}{l}\text { bacias sobre crosta oceânica sem espalhamento ou subducção associados (sem } \\
\text { bordas de placa ativas) }\end{array}$ \\
\hline \multirow{10}{*}{$\begin{array}{l}\text { AMBIENTE } \\
\text { CONVERGENTE }\end{array}$} & fossas & calhas profundas formadas pela subducção de litosfera oceânica \\
\hline & bacias talude-fossa & depressões estruturais localizadas, desenvolvidas em complexos de subducção \\
\hline & bacias de antearco & bacias entre a fossa e o arco \\
\hline & bacias de intra-arco & bacias na plataforma do arco \\
\hline & bacias de retroarco & $\begin{array}{c}\text { bacias oceânicas atrás de arcos magmáticos intra-oceânicos (incluindo bacias } \\
\text { inter-arco entre arcos ativos e remanescentes), e bacias continentais atrás de } \\
\text { arcos magmáticos de margem continental sem cinturôes de } \\
\text { dobramento/cavalgamento de foreland }\end{array}$ \\
\hline & $\begin{array}{l}\text { bacias foreland de } \\
\text { retroarco }\end{array}$ & $\begin{array}{l}\text { bacias foreland no lado continental de margens continentais associadas a } \\
\text { sistemas de arco-fossa, formadas por compressão relativa a subducção e/ou } \\
\text { colisão }\end{array}$ \\
\hline & $\begin{array}{l}\text { bacias oceânicas } \\
\text { remanescentes }\end{array}$ & $\begin{array}{l}\text { bacias oceânicas capturadas e confinadas entre margens continentais em } \\
\text { colisão e/ou sistemas arco-fossa, em processo final de subduç̧ão ou } \\
\text { deformação em zonas de sutura }\end{array}$ \\
\hline & bacias foreland periféricas & $\begin{array}{l}\text { bacias for eland desenvolvidas a partir de margens continentais rifteadas e } \\
\text { afetadas por zonas de subduç̧ão durante colisôes crustais (tipo primário de } \\
\text { foreland associado a colisão) }\end{array}$ \\
\hline & bacias piggyback & bacias formadas e alçadas por frentes cavalgantes \\
\hline & $\begin{array}{l}\text { foreland intermontanas } \\
\text { (foreland fragmentadas) }\end{array}$ & bacias foreland formadas em soerguimentos com embasamento envolvido \\
\hline \multirow{3}{*}{$\begin{array}{l}\text { AMBIENTE } \\
\text { TRANSFORMANTE }\end{array}$} & bacias transtracionais & bacias formadas por extensão em sistemas transcorrentes \\
\hline & bacias transpressionais & bacias formadas por compressão em sistemas transcorrentes \\
\hline & bacias transrotacionais & $\begin{array}{l}\text { bacias em sistemas transcorrentes formadas pela rotação de blocos crustais } \\
\text { segundo eixos verticais }\end{array}$ \\
\hline \multirow{4}{*}{$\begin{array}{l}\text { AMBIENTE } \\
\text { HÍBRIDO }\end{array}$} & $\begin{array}{l}\text { bacias wrench } \\
\text { intracontinentais }\end{array}$ & $\begin{array}{l}\text { bacias formadas em crosta continental como resposta distal a processos } \\
\text { colisionais }\end{array}$ \\
\hline & aulacógenos & $\begin{array}{l}\text { riftes abortados em alto ângulo com margens continentais, que foram } \\
\text { posteriormente reativados durante tectônica convergente }\end{array}$ \\
\hline & impactógenos & $\begin{array}{l}\text { riftes formados em alto ângulo com orógenos, sem história pré-orogenética } \\
\text { (em contraste com aulacógenos) }\end{array}$ \\
\hline & bacias sucessoras & $\begin{array}{c}\text { bacias formadas em ambientes intermontanos, após cessação de atividade } \\
\text { orogenética ou tafrogenética }\end{array}$ \\
\hline
\end{tabular}


mos, a saber: (i) puramente termais, (ii) envolvendo mudanças na espessura crustal/litosférica e (iii) envolvendo a adição ou retirada de sobrecarga. Assim, Allen \& Allen (1990) agruparam as bacias sedimentares de acordo com o esquema mostrado na Tabela 10.

DISCUSSÃO Pelo exposto acima, fica clara a grande dificuldade envolvendo qualquer tentativa de ordenar sistematicamente e classificar de forma coerente qualquer campo do conhecimento, na medida em que envolve a adoção de simples palavras ou frases para expressar definições ou conceitos complexos. $\mathrm{O}$ caso particular das bacias sedimentares é ainda mais complexo, na medida em que envolve entidades dinâmicas, com trajetórias evolutivas complexas e, muitas vezes, permeadas por profundas alterações nos mecanismos genéticos que governam sua evolução. O resultado disto é o grande número de categorias observado em diversos esquemas classificatórios. Como enfatizado por Dickinson (1993), o reconhecimento da diversidade envolvendo a classificação de bacias tem sido uma importante contribuição, mas, com isso, o número de tipos genéricos de bacias tende a aumentar na medida em que aprendemos mais sobre bacias individuais. Outro problema apontado por Dickinson (1993) refere-se à abordagem corrente da tipologia de bacias muitas vezes baseada em uma análise por analogia.

Outro aspecto que em muito dificulta a concepção de um esquema amplamente satisfatório para classificar bacias sedimentares é a dificuldade em se definir critérios genéticos, essencialmente interpretativos (sobretudo mecanismos de subsidência), bem como o posicionamento da bacia em relação ao posicionamento relativo à(s) placa(s) tectônica(s) envolvida(s), possibilitando a caracterização e a individualização de ciclos de $1^{a}$ ordem.

O exemplo clássico para este tipo de problema é a categoria denominada de "bacias intracratônicas". O que seria uma bacia intracratônica? Seria simplesmente uma bacia desenvolvida em litosfera continental sobre um substrato cratônico? O regime tectônico, o campo de tensões predominante e/ou mecanismo principal de subsidência devem ser levados em consideração na definição? Poderiam ser explicadas em termos de processos de margem de placa? Não seriam uma panacéia de casos "mal resolvidos"? Um análise da literatura sobre bacias intracratônicas mostra que, nesta categoria, estão englobados diversos tipos de bacias, se critérios genéticos forem levados em consideração. Klein (1995) enumera onze hipóteses propostas na literatura para explicar a origem de bacias intracratônicas. Muitas destas bacias têm sido recentemente, à luz de novos dados, reinterpreta- das com base em critérios genéticos ou geodinâmicos. Quilan \& Beaumont (1984), por exemplo, interpretam a origem das clássicas bacias "intracratônicas" Michigan e Illinois, EUA, como produto de flexura devido à Orogênese Apalachiana, reclassificando-as como bacias do tipo antepaís (foreland). Milani (1992, 1997), Milani \& Ramos (1998), Assine et al. (1998) e Assine (2001) da mesma forma consideram a deposição da seção paleozóica-eotriássica da Bacia do Paraná em um contexto de bacia de antepaís, sugerindo que os ciclos transgressivo-regressivos e o desenvolvimento das seqüências e descontinuidades regionais seriam resultantes de megaffexuras associadas a eventos colisionais na margem meridional do supercontinente Gondwana, associados a ciclos glacio-eustáticos. Verdadeiras bacias intracratônicas, ou intracontinentais na terminologia de Dickinson (1974), seriam bacias desenvolvidas no interior de placas continentais, devendo-se ao resfriamento de plumas mantélicas. As sinéclises do norte Africano (p.ex., bacias Taoudenni, Murzuk e Kufra, Moussine-Pouchkine \& Bertrand-Sarfati 1997, Lüning et al. 2000, Sola \& Worsley 2000, Hallett 2002), bem como a Bacia Canning na Austrália (Bender 2000) seriam exemplos deste tipo de bacia.

Por outro lado, a dinâmica evolutiva das bacias, manifestada através da atuação de sucessivos regimes de subsidência e da mudança de sua posição (no espaço e no tempo) em relação às placas tectônicas, cria uma dificuldade extra, na medida em que impõe a "passagem" de uma entidade bacinal por diferentes categorias dos esquemas classificatórios. Assim, adotando-se por exemplo os princípios de Dickinson (1974), uma bacia de margem continental do tipo atlântico (Bacia de Campos, por exemplo), embora seja considerada como uma única bacia sedimentar, possui uma fase ou etapa ligada a um ambiente divergente e outra ligada a um ambiente intraplaca. Sobre este aspecto, o esquema de Allen \& Allen (1990) se mostra mais eficiente, na medida em que toda a bacia pode ser enquadrada em uma única categoria (no caso da Bacia de Campos, bacias devido a estiramento ou afinamento litosférico), permitindo a conexão lógica de processos genéticos no tempo.

Perspectivas futuras $O$ exposto acima indica a necessidade de uma abordagem dinâmica para a classificação de bacias sedimentares, ou seja, mostra que as bacias sedimentares devem ser encaradas como entidades que, na maior parte dos casos, percorrem uma trajetória evolutiva através de diferentes ambientes geotectônicos, sendo normalmente submetidas a diferentes mecanismos de subsidência, que podem agir simultaneamente ou

Tabela 10: Classificação de bacias conforme Allen \& Allen (1990).

\begin{tabular}{|c|c|c|}
\hline CATEGORIAS & TIPOS & MECANISMOS \\
\hline $\begin{array}{l}\text { BACIAS DEVIDO A ESTIRAMENTO } \\
\text { OU AFINAMENTO LITOSFÉRICO }\end{array}$ & $\begin{array}{c}\text { suite rifte-drift de bacias (sags, riftes, } \\
\text { riftes abortados, aulacógenos e } \\
\text { margens passivas) }\end{array}$ & $\begin{array}{l}\text { comportamento termal e mecânico da } \\
\text { litosfera sob tensão e contração termal } \\
\text { da litosfera pós-estiramento }\end{array}$ \\
\hline \multirow{2}{*}{$\begin{array}{c}\text { BACIAS GERADAS POR FLEXURA } \\
\text { DA LITOSFERA CONTINENTAL OU } \\
\text { OCEÂNICA }\end{array}$} & $\begin{array}{l}\text { fossas na litosfera oceânica geradas na } \\
\text { aproximação de zonas de subducção }\end{array}$ & $\begin{array}{l}\text { combinação de forças gravitacionais na } \\
\text { litosfera oceânica em subducção e } \\
\text { excesso de massa do arco magmático }\end{array}$ \\
\hline & $\begin{array}{l}\text { bacias foreland em zonas de colisão } \\
\text { continental }\end{array}$ & $\begin{array}{c}\text { sobrecarga representada pela cadeia de } \\
\text { montanhas }\end{array}$ \\
\hline $\begin{array}{l}\text { BACIAS TRANSCORRENTES OU } \\
\text { RELACIONADAS A } \\
\text { MEGACISALHAMENTOS }\end{array}$ & bacias pull-apart & $\begin{array}{l}\text { envolve rotação de blocos crustais ou } \\
\text { extensão crustal thin-skinned }\end{array}$ \\
\hline
\end{tabular}


serialmente, na medida em que a bacia evolui.

Dickinson (1993) aponta para a necessidade do abandono de uma "taxonomia quasi-estática" para bacias sedimentares, onde seus processos formadores são inferidos como uma função derivada do tipo de bacia, enfatizando que devemos caminhar em direção a uma taxonomia mais dinâmica e flexível. De acordo com Dickinson (1993), categorias de bacias devem ser vistas como uma função complexa de processos mistos, operativos em graus variados e em combinações e seqüências diversas em diferentes ambientes tectônicos. Desta forma, este autor sugere a necessidade de se erigir uma classificação de bacias focada nos seus processos formadores fundamentais, que poderiam ser agrupados de diferentes maneiras para definir espectros de bacias, abandonando os "catálogos de tipos de bacias". Uma entidade bacinal seria então tratada em termos da associação de processos que governaram sua evolução e não como uma variedade de um tipo ideal.

De acordo com esta abordagem, os mecanismos de subsidência seriam os elementos chave na formação de bacias. Assim, de acordo com Dickinson (1993), uma abordagem geodinâmica para classificar bacias partiria da existência de um número limitado de processos que causam e controlam a subsidência em bacias, sendo que estes mecanismos podem combinar-se em diferentes proporções, no espaço e no tempo, e variar significativamente nas suas magnitudes. Conseqüentemente, mesmo tendo cada bacia suas peculiaridades próprias, elas podem ser descritas e classificadas em termos da combinação dos mecanismos de subsidência. De acordo com esta abordagem, torna-se necessária uma análise ponderada dos diferentes mecanismos de subsidência que controlaram a evolução da bacia, no sentido de relacionar diferentes arcabouços estratigráficos e histórias termais com diferentes padrões de combinação e seqüenciamento de mecanismos. Assim, cada bacia passaria a ser vista como o produto de uma combinação de processos controladores (mecanismos de subsidência) e não como variedades de uma determinada categoria (Dickinson, 1993).

Entretanto, na medida em que bacias sedimentares evoluem em resposta a uma complexa interação de processos mistos, operativos em graus variados e em combinações e seqüências diversas em diferentes ambientes tectônicos, a concepção de um esquema amplamente satisfatório para classificar bacias sedimentares esbarra na dificuldade prática da definição segura de critérios genéticos essencialmente interpretativos, como o(s) mecanismo(s) de subsidência atuante(s) na evolução de uma determinada bacia.

CONCLUSÕES Pelo exposto acima e, sobretudo, tendo em vista a vasta literatura sobre bacias sedimentares, conclui-se que os critérios básicos propostos por Dickinson (1974) (Tabela 5), embora incompletos e/ou deficientes sobre alguns aspectos, se mostraram bastante eficientes do ponto de vista prático, sobretudo ao enfatizarem o tipo de interação de placas ativo (regime tectônico) durante a sedimentação (se divergente, convergente ou transcorrente). $\mathrm{O}$ fato da evolução de bacias poder ser explicada com base nas mudanças nas relações de interação de placas resolveu o problema maior dos esquemas anteriores, que era a proliferação de nomes para satisfazer casos particulares.

Do ponto de vista da aplicação do esquema, o critério básico baseado no regime tectônico possuí implicações diretas em termos de campos de tensão, estilos estruturais e estilos estratigráficos, conferindo uma eficiência prática na definição do tipo de bacia e um caráter preditivo em termos de processos e produtos, muito útil em atividades exploratórias.

A literatura mostra que, embora critérios mais coerentes em termos do uso e entendimento da combinação de processos formadores de bacias e que satisfazem todo o espectro de bacias sem enfatizar apenas os end members, como o de Allen \& Allen (1990) (tabela 10), tenham sido propostos, as categorias básicas e a terminologia de Dickinson (1974) norteiam a maioria dos artigos e compêndios subseqüentes sobre o tema (p. ex., Frostick \& Steel 1993, Hancock 1994, Busby \& Ingersoll 1995), sendo amplamente utilizadas pela comunidade acadêmica e pela indústria do petróleo. Ademais, esquemas classificatórios posteriores a Dickinson (1974) que surgiram como tentativa de aperfeiçoamento deste (p.ex., Miall 1984, 1990, Ingersoll 1988 e Ingersoll \& Busby 1995) pecam em criar um número excessivo de categorias para classificar estágios de uma evolução contínua ou para satisfazer casos particulares, não tendo sido assimilados pela comunidade.

A Tabela 11 mostra as pequenas modificações e/ou atualizações em relação à proposta original de Dickinson (1974), sintetizadas da literatura e, sobretudo, fiéis à aplicação consagrada pela comunidade, destacando-se:

(i) o deslocamento dos riftes e riftes abortados da categoria de bacias intraplaca para bacias associadas a placas divergentes, enfatizando o regime tectônico em detrimento do substrato;

(ii) exclusão da categoria intraplaca em substrato transicional (herdado de antigas junções de placas), e incorporação do consagrado termo "bacias de margem passiva", uma vez que bacias desta natureza representam a fase flexural da evolução de bacias em regime divergente, sendo assim denominadas e, de forma simplificada, enquadradas nesta categoria;

(iii) adoção do termo "transcorrente (ou strike-slip, em inglês)" no lugar de "transformante", uma vez que o último implica necessariamente no envolvimento de crosta oceânica, sendo um caso particular do primeiro;

(iv) incorporação das bacias "pull-apart" dentro das transcorrentes envolvendo o cisalhamento intracontinental e "margens transformantes" envolvendo cisalhamento de litosferas continental e oceânica.

Agradecimentos Este artigo é fruto de muitos anos de trabalho em diferentes bacias sedimentares, onde o autor se deparou inúmeras vezes com o dilema de se classificar bacias. Desta forma, o autor expressa seus agradecimentos a muitos colegas com os quais compartilhou discussões frutíferas sobre o tema, sobretudo a Fernando Alkmim (Ufop), Frank Falkenhein (Petrobras) e Pat Eriksson (Univ. Pretória). Ao CNPq pela concessão de Bolsa de Produtividade em Pesquisa ao autor, sempre vinculada a estudos em bacias sedimentares, e à FAPEMIG por auxílios pesquisa em projetos focando bacias. Aos revisores da RBG pelas valiosas contribuições. Finalmente, à interação universidade-indústria (em especial devida à Ufop, Fundação Gorceix e Petrobras) que possibilita um saudável e prolífero intercâmbio de experiências e o acesso a dados sísmicos, normalmente inacessíveis à universidade e essenciais à compreensão do complexo universo das bacias sedimentares. 
Tabela 11: Classificação de bacias, modificada/atualizada de Dickinson (1974).

\begin{tabular}{|c|c|c|}
\hline \multirow{2}{*}{ INTRAPLACA } & \multicolumn{2}{|r|}{ intracontinentais* (substrato continental) } \\
\hline & \multicolumn{2}{|r|}{ bacias oceânicas profundas (substrato oceânico) } \\
\hline \multirow{6}{*}{$\begin{array}{c}\text { ZONAS } \\
\text { (REGIMES) DE } \\
\text { INTERAÇÃO } \\
\text { ENTRE PLACAS }\end{array}$} & \multirow{2}{*}{ DIVERGENTE } & riftes e riftes abortados \\
\hline & & proto-oceanos e margens passivas \\
\hline & \multirow[t]{2}{*}{ CONVERGENTE } & $\begin{array}{c}\text { sistema arco/fossa (bacias de fossa, antearco, intra-arco, } \\
\text { inter-arco, retroarco, antepaís de retroarco) }\end{array}$ \\
\hline & & bacias de antepaís periféricas a colisões continentais \\
\hline & \multirow{2}{*}{ TRANSCORRENTE } & bacias pull-apart (continental-continental) \\
\hline & & margens transformantes (continental-oceânica) \\
\hline
\end{tabular}

\section{Referências}

Allen P.A. \& Allen J.R. 1990. Basin Analysis: Principles and Applications. Blackwell, Oxford, 45lpp.

Assine, M.L. 2001. O ciclo devoniano na Bacia do Paraná e correlação com outras bacias Gondwânicas. In: Melo, J.H.G \& Terra, G.J.S. (eds.) Correlação de Seqüências Paleozóicas Sul-americanas. Série Ciência-Técnica-Petróleo, Seção Exploração de Petróleo, 20: 55 62.

Assine, M.L.; Perinotto, J.A.J.; Alvarenga, C.J.S.; Petri, S. 1998. Arquitetura estratigráfica, tratos deposicionais e paleogeografia da Bacia do Paraná (Brasil) no Neo-Ordovinciano/Eo-Siluriano. Revista Brasileira de Geociências, 28: 61-76.

Bally A.W. \& Snelson S. 1980. Realms of subsidence. Can. Soc. Petrol. Geol., Memoir 6: 9-75.

Bender, A., 2000. Mechanisms of intracratonic and rift basin formation: Insights from the Canning basin, northwest Australia. Lamont-Doherty Earth Observatory of Columbia University, New York, Ph.D. Thesis (unpublished).

Busby C.J. \& Ingersoll R.V. 1995. Tectonics of Sedimentary Basins. Blackwell, Oxford, 579pp.

Dana D.J. 1866. Observations on the origin of some of the Earth's features. American J. Sci., 2: 205-211.

Dana D.J. 1873. On some results of Earth's contractions from cooling, including a discussion of the origin of mountains and the nature of Earth's interior. American J. Sci., ser. 3, 5: 423-453, 6: 6-14, 104-115, 161-172.

Dewey J.F. 1969. Continental margins: a model for the transition from Atlantic type to Andean type. Earth and Planetary Sci. Letters, 6: 189-197.

Dewey J.F. \& Bird J.M. 1970. Mountain belts and the new global tectonics. $J$. Geophys. Res., 75: 2625-2647.

Dias J.L., Scarton, J.C., Guardado, L.R., Esteves F.R. \& Carminatti M. 1990. Aspectos da evolução tectono-sedimentar e a ocorrência de hidrocarbonetos na Bacia de Campos. In: G.P. Raja Gabaglia \& E.J.
Milani (eds.) Origem e Evolução de Bacias Sedimentares, Petrobras, pp.: 333-360.

Dickinson W.R. 1971a. Plate tectonic models for orogeny at continental margins. Nature, 232: 41-42.

Dickinson W.R. 1971b. Plate tectonics in geologic history. Science, 174 107-113.

Dickinson W.R. 1971c. Plate tectonic models of geosynclines. Earth and Planetary Sci. Letters, 10: 165-174.

Dickinson W.R. 1974. Plate tectonics and sedimentation. In: W.R. Dickinson (ed.) Tectonics and Sedimentation, SEPM, Spec. Publ., 22: $1-27$.

Dickinson W.R. 1993. Basin geodynamics. Basin Res., 5: 195-196.

Dietz R.S. 1961. Continent and ocean basin evolution by spreading of the sea floor. Nature, 190: 854-857.

Dietz R.S. 1963. Collapsing continental rises, an actualistic concept of geosynclines and mountain building. J. Geol., 71: 314-333.

Drake C.L. 1966. Recente investigations on the continental margin of eastern United States. In: W.H. Poole (ed.) Continental Margins and Island Arcs. Geol. Survey Canada Paper 66-15, pp. 33-47.

Eriksson P.G., Martins-Neto M.A., Nelson D.R., Aspler L.B., Chiarenzelli J.R., Catuneanu O., Sarkar S. \& Rautenbach C.J. de W. 2001 a. An introduction to Precambrian basins: character, classification and major genetic influences. In: P.G. Eriksson, M.A. Martins-Neto, O. Catuneanu, L. Aspler \& J. Chiarenzelli (eds.) The influence of magmatism, tectonics, sea level change and palaeoclimate on Precambrian basin evolution: change over time, Sedimentary Geology Spec. Issue, 141-142: 1-35.

Eriksson P.G., Catuneanu O., Aspler L.B., Chiarenzelli J.R. \& MartinsNeto M.A.- editores $2001 \mathrm{~b}$. The influence of magmatism, tectonics, sea level change and palaeoclimate on Precambrian basin evolution: change over time. Sedimentary Geology Spec. Issue, vol. 141$142,522 \mathrm{pp}$. 
Fedo C.M., Myers J.S. \& Appel P.W.U. 2001. Depositional setting and paleogeographic implications of Earth's oldest supracrustal rocks, the $>3.7$ Ga Isua Greenstone belt, West Greenland. In: P.G. Eriksson, M.A. Martins-Neto, O. Catuneanu, L. Aspler \& J. Chiarenzelli (eds.) The influence of magmatism, tectonics, sea level change and palaeoclimate on Precambrian basin evolution: change over time, Sedimentary Geology Spec. Issue, 141-142: 61-77.

Figueiredo A.M.F. \& Raja Gabaglia G.P. 1986. Sistema classificatório aplicado às bacias sedimentares brasileiras. Rev. Bras. de Geoc., 16: 350-369.

Frostick L. E. \& Steel R.J. - editores 1993. Tectonic Controls and Signatures in Sedimentary Successions. Intern. Assoc. Sediment., Spec. Publ. 20, 520pp.

Hall J. 1859. Description and figures of the organic remains for the lower Helderberg Group and the Oriskany Sandstone. Geol. Survey Albany, New York, 544pp.

Hallett D. 2002. Petroleum Geology of Lybia. Elsevier, Amsterdam, $508 \mathrm{pp}$.

Hancock P.L. 1994. Continental Deformation. Pergamon Press, Oxford, $421 \mathrm{p}$.

Haug E. 1900. Les géosynclinaux et les aires continentales. Contribution à l'etude des regressions et des transgressions marines. Soc. Géol. France, Bull., 28: 617-711.

Hsü K.J. 1965. Collapsing continental rises: an actualistic concept of geosynclines and mountain building: a discussion. J. Geol., 73: 897900.

Ingersoll R.V. 1988. Tectonics of sedimentary basins. Geol. Soc. Am., Bull., 100: 1704-1719.

Ingersoll R.V. \& Busby C.J. 1995. Tectonics of sedimentary basins. In: C.J. Busby \& R.V. Ingersoll (eds.) Tectonics of Sedimentary Basins. Blackwell, Oxford, pp: 1-51.

Kay M. 1947. Geosynclinal nomenclature and the craton. AAPG Bull., 31: 1289-1293.

Kay M. 1951. North American geosynclines. GSA Memoir 48: 143pp.

Kingston D.R., Dishroon, C.P. \& Williams, P.A. 1983. Global basin classification. AAPG Bull., 67: 2175-2193.

Klein G.D. 1995. Intracratonic basins. In: C.J. Busby \& R.V. Ingersoll (eds.) Tectonics of Sedimentary Basins. Blackwell, Oxford, pp: 459478.

Klemme H.D. 1980. Petroleum basins - classification and characteristics. J. Petrol. Geol., 3: 187-207.

Krapez B. 1993. Sequence stratigraphy of the Archean supracrustal belts of the Pilbara block, Western Australia. Precambrian Res., 60: 1-45.

Laughton A.S. 1966. The Gulf of Aden, in relation to the Red Sea and the Afar Depression of Ethiopia. Can. Geol. Survey Paper, 66-14: 78-97.

Lüning S., Craig J., Fitches W.R., Gammudi A., Mayouf J., Busrewil A., El Dieb M. \& Loydell D. K. 2000. Petroleum source rock-reevaluation of the Kufra Basin (SE Libya, NE Chad). In: Sola M. A. \& Worsley D. (eds.), Geological Exploration in Murzuq Basin, Elsevier, Amsterdam, pp. 151-173.

Martins-Neto 1998. O Supergrupo Espinhaço em Minas Gerais: Registro de uma bacia rifte-sag do Paleo/Mesoproterozóico. Rev. Bras. de Geoc., 28: 151-168.
Martins-Neto M.A. 2000. Tectonics and sedimentation in a Paleo/Mesoproterozoic rift-sag basin (Espinhaço Basin, southeastern Brazil). Precambrian Res., 103: 147-173.

Martins-Neto, M.A. 2005. A Bacia do São Francisco: arcabouços estratigráfico e estrutural com base na interpretação de dados de superfície e subsuperficie. In: SBG, Simp. Craton São Francisco, 3, Salvador, Anais, 283-286.

Martins-Neto M.A. \& Alkmim F.F. 2001. Estratigrafia e evolução tectônica das bacias neoproterozóicas do paleocontinente São Francisco e suas margens: registro da quebra de Rodinia e colagem de Gondwana. In: C.P. Pinto \& M.A. Martins-Neto (eds.), Bacia do São Francisco: Geologia e Recursos Naturais, Publicação Especial da SBG-MG, pp. 31-54

Martins-Neto M.A., Pedrosa-Soares A.C., Lima S.A.A. 2001. Tectonosedimentary evolution of sedimentary basins from Late Paleoproterozoic to Late Neoproterozoic in the São Francisco craton and Araçuai fold belt, eastern Brazil. In: P.G. Eriksson, M.A. Martins-Neto, O. Catuneanu, L. Aspler \& J. Chiarenzelli (eds.) The influence of magmatism, tectonics, sea level change and palaeoclimate on Precambrian basin evolution: change over time, Sedimentary Geology Spec. Issue, 141-142: 343-370.

Menard H.W. 1967. Transitional types of crust under small ocean basins. J. Geophys. Res., 12: 3061-3073.

Miall A.D. 1984. Principles of Sedimentary Basin Analysis. 1" edição, Springer-Verlag, New York, 490p.

Miall A.D. 1990. Principles of Sedimentary Basin Analysis. $2^{a}$ edição, Springer-Verlag, New York, 668p.

Milani E.J. 1992. Intraplate tectonics and the evolution of the Paraná basin. In: M.J. De Wit \& I.G.D. Ransome (eds.) Inversion Tectonics of the Cape Fold Belt, Karoo and Cretaceous Basins of Southern Africa, Balkema, Rotterdam, pp. 101-108.

Milani E.J. 1997. Evolução tectono-estratigráfica da Bacia do Paraná e seu relacionamento com a geodinâmica fanerozóica do Gondwana sul-ocidental. Instituto de Geociências. Tese de Doutoramento, Universidade Federal do Rio Grande do Sul, $255 \mathrm{p}$.

Milani, E.J. \& Ramos, V.A. 1998. Orogenias paleozóicas no domínio sul-ocidental do Gondwana e os ciclos de subsidência na Bacia do Paraná. Revista Brasileira de Geociências, 28: 527-544.

Mitchell A.H.G. \& Reading H.G. 1969. Continental margins, geosynclines and ocen floor spreading. J. Geol., 77: 629-646.

Moussine-Pouchkine A. \& Bertrand-Sarfati J. 1997. Tectonosedimentary subdivisions in the Neoproterozoic to Early Cambrian cover of the Taoudenni Basin (Algeria-Mauritania-Mali). J. African Earth Sci., 24: $425-443$

Quilan G.M. \& Beaumont C. 1984. Appalachian thrusting, lithospheric flexure, and the Paleozoic stratigraphy of the eastern interior of North America. Can. J. Earth Sci., 21: 973-996.

Sola M.A. \& Worsley D. eds. 2000. Geological exploration in Murzuq Basin. Elsevier, Amsterdam, 519 pp.

Stille H. 1940. Einführung in den Bau Nordamerikas. Bornträger, Berlim, $717 \mathrm{pp}$.

Weeks L.G. 1952. Factors of sedimentary basin development that control oil occurrence. AAPG Bull., 36: 2071-2124.

Manuserito A-1573

Revisão aceita em 14 de junho de 2006 